\title{
Improving the quality of hospital discharge summaries utilising an electronic prompting system
}

\author{
Andrew P Maurice, Samuel Chan, Clifford W Pollard, Richard A Kidd, Stephen J Ayre, Helen E Ward, Darren L Walters \\ The Prince Charles Hospital, Brisbane, Queensland, Australia
}

\begin{abstract}
The discharge summary (DS) is a summary of an inpatient admission, patient's health state, and future treatment plans which is delivered to the patient's primary care provider. The DS is often incomplete, inaccurate, or unclear. The aim of this project was to improve the quality of the DS through the use of an electronic prompting system. The electronic prompting system was implemented in the acute medical and surgical wards of the hospital as an adjunct to a pre-existing, widely used hospital program that documents all the patients in a ward or belonging to a particular treating team. When using the program, a doctor enters information (with the assistance of the treating consultant) from a drop-down menu and is prompted to include common, departmental specific diagnoses, co-morbidities, complications, and procedures that were commonly missed or documented incorrectly in the DS.
\end{abstract}

Fifteen DSs were randomly selected from a two month period immediately prior to the intervention period and were rated by an external, experienced general practitioner (GP) using a scoring system consistent with the Australian Medical Association Guidelines for quality DSs. Fifteen random DSs from a two month period, four months post-implementation were also rated by the same GP.

The quality of the DS improved in all categories evaluated. The overall quality improved from mean ( \pm SD) $2.86 \pm 1.64$ to $4.13 \pm 0.92$ out of 5 $(p=0.031)$. Additionally the implementation of the system was associated with improvements in documentation of the diagnosis, comorbidities and other relevant clinical information.

In summary, electronic prompting systems can improve the quality of DSs to ensure the information contained within the DS is more accurate and complete.

\section{Problem}

Numerous studies have demonstrated that discharge summaries (DSs) are often inaccurate. A prospective eight month audit of a vascular surgery unit demonstrated that $22 \%$ of diagnoses were incorrect, $19 \%$ incorrectly recorded the procedure, $19 \%$ contained clinically incorrect information, $14 \%$ did not document significant complications, and $11 \%$ had incorrect follow-up arrangements.[1] An Australian study demonstrated that up to $36.4 \%$ of DS in their health service contained errors.[2] Another recent Australian study demonstrated that up to $10 \%$ of the principal diagnoses in the DS were incorrect.[3] Errors in the patient's DS have been strongly correlated to increased re-admission rates to hospital and adverse patient events.[4,5]

In light of these statistics, it is alarming that the diagnosis and comorbidities are not correctly recorded in the DS, especially if the primary care physician (in Australia, a GP) has to make treatment and follow-up plans based on the DS. For instance, a patient admitted with an acute coronary syndrome may also be diagnosed with hypertension, dyslipidaemia, and type II diabetes mellitus as an inpatient. If this patient visits their GP for review after discharge, it is vital the GP is informed about these co-morbidities as they will each individually require follow up and further treatment, generally arranged by the GP.
Furthermore, it is also vital to correctly document complications such as blood loss, wound infection, and electrolyte abnormalities that require follow up. It is also important that any procedures undertaken in the hospital setting are documented correctly. For instance, a patient who has received a drug-eluting coronary artery stent will typically require two anti-platelet agents for one year to prevent catastrophic thrombosis of the stent. It is vital such information is communicated to the GP to ensure these agents are not discontinued inadvertently.

The literature suggests that errors and omissions are frequent when recording the diagnosis, procedure, co-morbidities or complications in a patient, thus affecting quality and safety of patient care. The aim of this project was to improve the quality of the DS utilising an electronic prompting system.

At our institution, we found that DS completion was a low priority for medical staff as direct patient care generally took precedence.[8] Furthermore, medical staff worked a considerable amount of overtime. Staff were also under considerable pressure to compile the DS within 48 hours of discharge.[8] It was felt that these factors adversely affect the quality of the DS.

\section{Background}

The Prince Charles Hospital (TPCH) is a 600 bed tertiary public 
hospital in Brisbane, Queensland, Australia, and provides services in The Heart and Lung Institute (cardio-thoracic medicine and surgery), general medicine, general surgery, orthopaedics, rehabilitation and acute stroke, geriatrics, palliative care and mental health. TPCH has approximately 3500 staff of which there are 277 junior and 143 senior medical staff.

When a patient is discharged from hospital, the medical team must complete a DS which outlines the details of the hospital admission as well as instructions for further care (figure 1). This document is forwarded to the primary care physician. At our institution, the DS is usually authored by the most junior medical staff, the resident medical officer (RMO), on behalf of the treating team and attending physician. The DS is compiled using an electronic computer program and automatically attaches the discharge medications.

When a DS is completed, the information is sent electronically or via post to the GP. At TPCH the DS is the only official means of communicating the inpatient treatment summary to the physician. Occasionally, the treating team will call the physician for a handover, though this is not mandatory.

The Australian Medical Association recommends that a DS should be produced for every patient by the hospital provider within 30 days of discharge and information should include[6]:

\section{- Reasons for hospitalisation}

- Procedures performed

- Care, treatment, and serves provided

\section{- Discharge condition}

- Information provided to the patient/family

- Accurate and up-to-date record of patient medications

- Attending physician signature.

\section{Baseline measurement}

A list of all DSs completed in a two-month period immediately prior to the intervention as well as for a two-month period, four months after the intervention was obtained for all acute medical and surgical departments within the hospital. This was accessed through the electronic discharge summary computer program available through the safety and quality unit at the hospital. Microsoft Excel was used to randomly select 15 DSs from the preand post-intervention period. These DSs were then provided to an experienced GP, external to our institution, to evaluate the quality of the DSs. To reduce bias, the patient's demographic details, the treating consultant's name, the author of the DS and date the DS was written was not made available to the assessor. The GP was aware of whether the DS was produced with or without the aid of the electronic prompting system.

The GP evaluated the DSs with a criteria sheet based on the AMA guidelines for an acceptable DS (figure 2).[6] This included the accuracy and clarity of the principal diagnosis, the clarity of the comorbidities, the procedures and the complications as well as the appropriateness of recommendations to the GP. The final part of the criteria sheet allowed the GP to give an overall quality score on a five point scale from "very poor" to "excellent".

\section{Design}

An electronic prompting system was utilised to assist with the entry of the correct information. Each acute surgical and medical department involved in the project developed a list of diagnoses, complications, co-morbiditiesm and procedures utilising the correct terminology consistent with the International Classification of Diseases Version 10 (Australian modification) classification.[7] Each day, a RMO entered the above information into a program, utilising drop down boxes to prompt them to document the diagnoses, complications, co-morbidities and procedures (figure 3), and these were reviewed and agreed by the treating consultant on the consultant ward round.

The RMO was able to enter free text into these entries, but the drop down menus contained the most relevant and often missed information. For instance, patients admitted into the coronary care unit would have "type II diabetes mellitus", "congestive cardiac failure", and "dyslipidaemia" in their diagnosis and co-morbidities prompting boxes. Similarly "post-operative blood loss treated with blood transfusion" and "hypokalaemia" were examples of prompts for complications. The precise prompts in the drop down menus were tailored to each department in the hospital.

To improve uptake and accessibility, the system was built into a preexisting program already present within the hospital. Specifically, this program was called the "Patient Electronic Journey Board" which displayed a list of the current inpatients in each ward of the hospital. This program was already widely used by both medical and allied health staff within the hospital to formulate patient lists for medical team ward rounds, determine which patients are admitted to which team as well as to document treatment plans for the nursing staff handovers.

Medical staff were able to use the information to automatically populate a patient lists to be used for ward rounds (figure 4). This replaced the time consuming RMO task of manually creating a patient list each day for ward rounds, thus creating an incentive for medical staff to use the program accurately. The automatic transfer of the information to the DS itself was also an incentive. This has been shown to reduce the time taken to complete a DS in a separate quality improvement project performed by our group.[8]

Information that was entered into the prompting system could also be printed and placed in the chart and used as ward round notes. The beneficial effects of this are being evaluated by our group in a separate quality improvement project.

\section{Strategy}


The electronic prompting system was developed by the hospital information technology department and was estimated to cost $\$ 40,000$ for implementation. Hospital IT staff assisted with modifying the current "Patient Electronic Journey Board" program to allow for the implementation of the prompting system. The project was approved by the hospital medical advisory committee as well as the financial department of the hospital. The project was supported by the executive director of medical services and the executive director of the hospital.

RMOs were given one hour training sessions in use of the program and were then encouraged by the departmental directors in the units to use the prompting system. Questions were answered and issues clarified by the authors of this study. Usage of this electronic prompting system was included in the unit orientation in the medical units in which the program was implemented.

PDSA cycle 1: This first cycle involved delivering the intervention to one unit in the hospital only. Problems that arose included poor understanding of the program and difficulties in accessing and using the program.

PDSA cycle 2: This involved providing more detailed orientation sessions as well as improving the user interface in conjunction with the IT department of the hospital. This helped alleviate many of the concerns encountered with the first cycle.

PDSA cycle 3: This involved implementing the project into other departments in the hospital. This required modification of the program to reflect terminology more appropriate to other units.

\section{Results}

The proportion of DS with adequate record of diagnosis, comorbidities, complications, inpatient care summary, and treatment as well as instructions for ongoing care of the GP all improved postintervention compared with pre-intervention (table 1). The number of DS that were "appropriate level" of detail increased significantly, however the number of DS with too much detail also increased. The number of DS with too little detail decreased.

Overall quality was subjectively marked as "very poor", "poor", "mediocre", "good", and "excellent". For sake of comparison, these were assigned scores from 1 to 5 and the improvement in scores from pre- to post-intervention is displayed in figure 5 . The overall quality improved significant from a mean of $2.86 \pm 1.64$ to $4.13 \pm$ 0.92 out of 5 (paired $t$ test: $p=0.031$ ).

Other information contained in the DS was not changed; in particular, there was no difference in the method of entering medications in the DS or of reconciliation of discharge medications by the pharmacist. One potential confounding factor in the interpretation of these results is that RMOs may have improved their skill and accuracy in completing DSs for a variety of uncontrolled reasons, including increased experience gained over the course of the academic year.

See supplementary file: ds3467.doc - "TABLES_AND_FIGURES"

\section{Lessons and limitations}

- At our institution, electronic department-specific prompts are useful in reminding medical staff to ensure that all patient diagnoses, co-morbidities, complications, and procedures are entered correctly, using appropriate and clear terminology

- The utilisation of an electronic prompting system to compile discharge summaries was associated with an improvement in the discharge summary quality and may improve the handover of patient information and reduce adverse patient outcomes

\section{Conclusion}

DS summaries are often of poor quality, often containing incorrect or incomplete documentation of patient diagnosis, co-morbidities, complications and procedures. An electronic prompting system can improve the quality of DSs and improve the handover of important clinical information to the GP and thus improve patient care.

\section{References}

1. Macaulay EM, Cooper GG, Engeset J, Naylor, AR. Prospective audit of discharge summary errors. Brit J Surg 1996; 788-90.

2. Wilson S, Ruscoe W, Chapman M, Miller R. General practitioner-hospital communications: a review of discharge summaries. J Qual Clin Pract 2001; 104-8.

3. Hospital Discharge Summary Audit. Eastern Health GP Liaison Team. https://www.easternhealth.org.au/app cmslib/media/umlib/g p/dischargeauditreporteh260412.pdf (retrieved March 31 2014).

4. Moore C, Wisnivesky J, Williams S, McGinn T. Medical errors related to discontinuity of care from an inpatient to an outpatient setting. J Gen Int Med 2003; 646-51.

5. O'Leary KJ, Liebovitz DM, Feinglass J, Liss DT, Baker DW. Outpatient physicians' satisfaction with discharge summaries and perceived need for an electronic discharge summary. J Hosp Med 1 2006: 317-20.

6. AMA Safe Handover: Safe Patients - Guidance on clinical handover for clinicians and managers. 2012, Australian Medical Association.

7. International Statistical Classification of Diseases and Related Health Problems, Tenth Revision, Australian Modification, 8th Edition, 2013.

8. Chan S, Maurice AP, Pollard CW, Ayre SJ, Walters DL, Ward HE. Improving the efficiency of discharge summary completion by linking to pre-existing patient information databases. BMJ Qual Improv Report. 2014; In press.

\section{Declaration of interests}

None declared. 


\section{Acknowledgements}

Nil acknowledgements 